\section{\$17. Development of Microwave Imaging Reflectometry in Large Helical Device}

Yoshinaga, T., Nagayama, Y.,

Kuwahara, D. (Tokyo Tech.), Shi, Z.B. (SOKENDAI), Yamaguchi, S. (Kansai Univ.),

Kogi, Y., Mase, A. (KASTEC, Kyushu Univ.)

In order to study various dynamic phenomena in plasmas such as transports or instabilities it is important to understand the spatial structures and of the related plasma parameters and the behavior of them. For the observation of the spatial structures of the electron density fluctuations microwave imaging reflectometry (MIR) has been developed in LHD. By focusing the scattered microwave which is reflected from the cutoff surface onto the twodimensional receiver antennas, MIR can reconstruct twodimensional structure of the cutoff surface. Therefore, MIR becomes quite sensitive to the local electron density near the cutoff surface. The final goal of the MIR is to reconstruct and visualize the density fluctuation structure in turbulence phenomena in LHD plasmas.

Figure 1 shows a schematic of the MIR system in LHD. The illumination wave $\left(\mathrm{f}_{\mathrm{RF}}=50-75 \mathrm{GHz}+0.11\right.$ $\mathrm{GHz}$ ) is projected into the plasma through the optics system which consists of several focusing $\mathrm{Al}$ mirrors and dielectric beam splitters. The direction of the illuminating wave injection can be adjusted to match the plasma shapes by changing the angle of the main mirror controlled by ultra-sonic-motors. The reflected microwaves which come back along the same optical path with the illumination wave are focused on the two-dimensional receiver antenna array, which consists of $5 \mathrm{ch}$ (toroidal) $\mathrm{x} 8 \mathrm{ch}$ (poloidal) small horn antennas [1]. The local waves $\left(f_{\mathrm{LO}}=50-75\right.$ $\mathrm{GHz}$ ) are also projected onto the receiver antenna array from the forward direction and mixed with the reflected waves by a mixer diode inside each small horn. The downconverted IF signals at $0.11 \mathrm{GHz}$ can be obtained by such super-heterodyne technique for the detection of the phases and the amplitudes.

Figure 2 shows a time evolution of the frequency spectrum of the reflected power observed by MIR. A fluctuation at $\mathrm{f} \sim 2.5 \mathrm{kHz}$ accompanied by several harmonic oscillations, which seems to be edge harmonic oscillation (EHO), are observed when the neutral beam injection is started at $\mathrm{t} \sim 1.4 \mathrm{~s}$. Magnetic measurements also showed the similar spectrum, confirming the MIR result. Considering that the illuminating wave is injected in $\mathrm{X}$ mode at $\mathrm{f}_{\mathrm{RF}} \sim 57 \mathrm{GHz}$, the cutoff surface is considered to be placed near the edge plasma region $(\rho \sim 0.9-1)$.

While the MIR system (especially the multi-channel receiver antenna system) was significantly improved and two-dimensional observation has been started in 2008, there remain some problems. It would be difficult to obtain a clear image of the cutoff surface motion at present because of the low $\mathrm{S} / \mathrm{N}$ ratio (less than 10) and some bad channels with quite low sensitivities. Improving these are the next issues.

1) Kuwahara, D. et al.: This annual report.

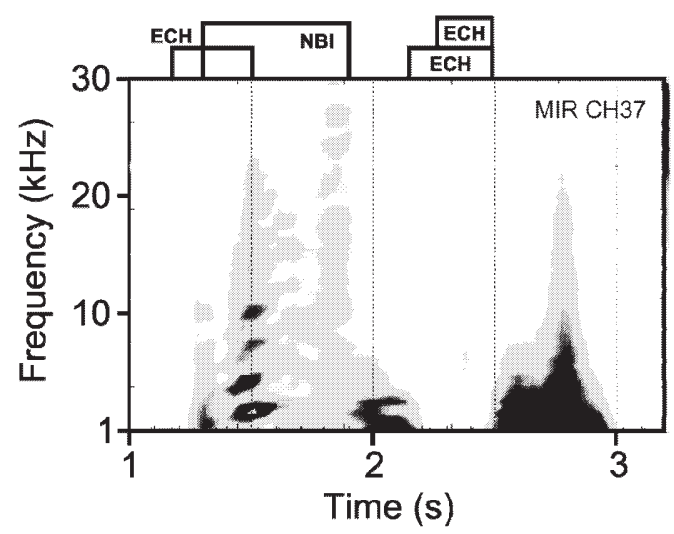

Fig. 2. The time evolution of the MIR spectrum. \#89643, $\mathrm{R}_{\mathrm{ax}}=3.6 \mathrm{~m}, \mathrm{Bt}=-2.75 \mathrm{~T}, \gamma=1.254, \mathrm{~Bq}=100 \%$.

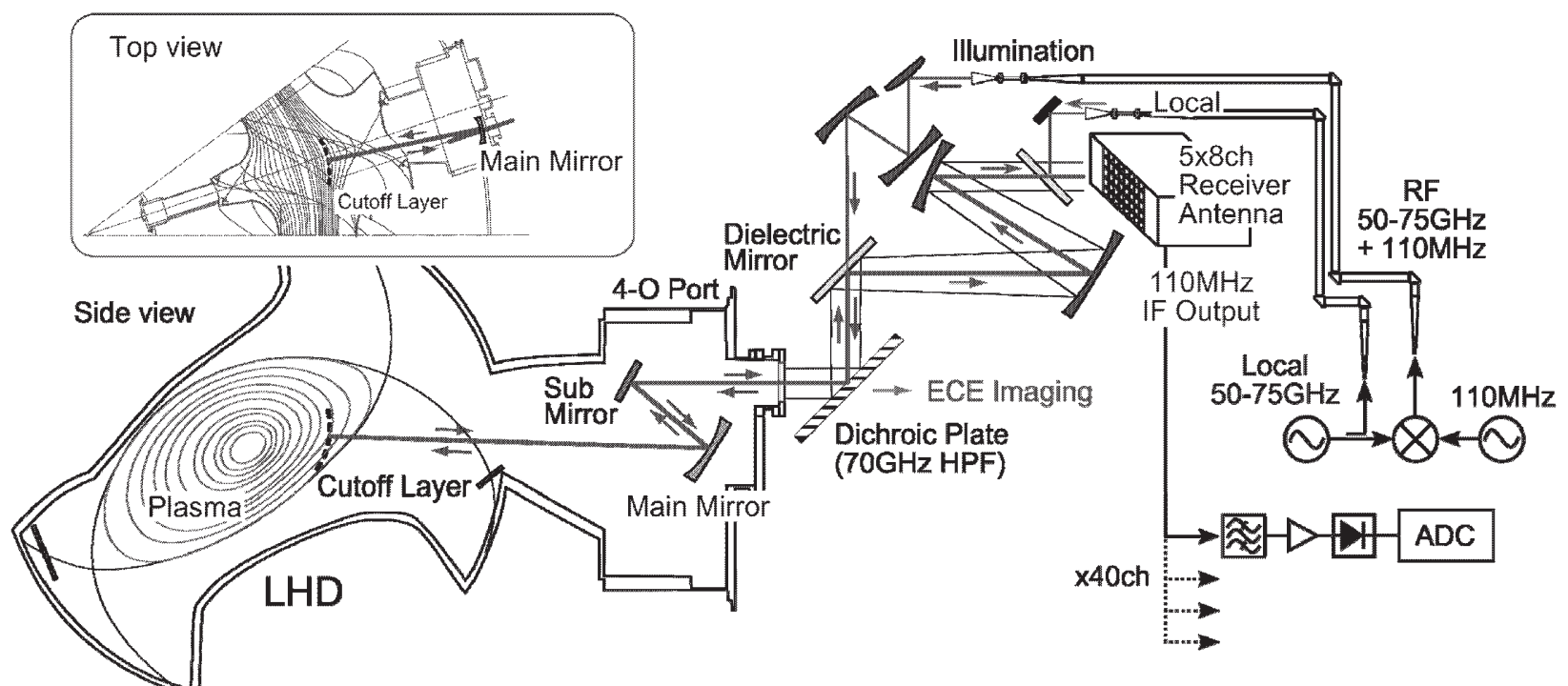

Fig. 1. Schematic view of the MIR system in LHD. 